\title{
Using advanced manufacturing technology for smarter construction
}

\author{
Allan Griffin MEng, PIEMA \\ Head of Construction and Strategy, Advanced Manufacturing Research \\ Centre, University of Sheffield, Sheffield, UK \\ Ruby Hughes BSC, PhD, CEng, MIMechE \\ Technical Fellow, Head of Manufacturing Intelligence Group, Advanced \\ Manufacturing Research Centre, University of Sheffield, Sheffield, UK \\ Chris Freeman MIET \\ Vice President for Industry 4.0, Sublime, Glasgow, UK \\ James Illingworth MEng \\ Theme Lead - Automated Assembly, Advanced Manufacturing Research \\ Centre, University of Sheffield, Sheffield, UK
}

\author{
Tomas Hodgson MSci, MIET \\ Theme Lead - Inspection and Al, Advanced Manufacturing Research \\ Centre, University of Sheffield, Sheffield, UK \\ Michael Lewis MEng \\ Theme Lead - Digital, Advanced Manufacturing Research Centre, \\ University of Sheffield, Sheffield, UK \\ Ejae Perez MEng \\ Senior Project Engineer, Advanced Manufacturing Research Centre, \\ University of Sheffield, Sheffield, UK
}

\begin{abstract}
Productivity in the UK construction sector has historically lagged behind other industry sectors. The government is aiming to improve this through increasing the level of pre-manufactured value in built assets. Since 2001, the University of Sheffield's Advanced Manufacturing Research Centre has been developing technological innovations for the aerospace and automotive sectors. This paper shows how lessons learnt from, and technologies developed for, these sectors can be transferred into the construction supply chain through horizontal innovation. Technologies such as robotics and automation, augmented and virtual reality, discrete event simulation, large volume metrology, and improved tools and processes all have a role to play. Significant productivity increases are possible, with the benefit often driven by the digitalisation of traditionally manual paper-based processes.
\end{abstract}

\section{Introduction}

The use of advanced manufacturing techniques developed and proved in high-value manufacturing sectors such as aerospace and defence may be thought to have little application for highvolume traditional sectors such as the construction industry. On the contrary, cascading technologies and production processes perfected in high-value manufacturing to construction is achievable, but is not without its challenges. Indeed, the biggest obstacle to a sector-wide transformation is less the adaptation of advanced technologies to construction (Brown, 2018) and more the reluctance among some industry leaders and policy makers to change a conservative mindset to one that embraces the adoption of industrial digitalisation: robotics; automation; augmented reality (AR), virtual reality (VR) and mixed reality; discrete event simulation (DES); and digital twins.

In the UK, it is recognised by industry leaders and policy makers within the construction sector that the current delivery model for built assets is broken and industry-wide change is required (Farmer, 2016; IPA, 2016, 2017). In the government's November 2017 budget, the chancellor announced a 'presumption in favour' of offsite construction by 2019 across suitable capital programmes, where it represents best value for money (HM Treasury, 2017). There is a growing consensus that a move towards offsite construction and greater pre-manufactured value will deliver clear and tangible benefits (HMG, 2018; SCST, 2018).

However, whereas the driver for change is clear, there is little understanding around how this could be achieved in practice or how technological innovation can play a role in delivering this ambition (HMG, 2018). This paper explores use cases for advanced manufacturing technologies in research projects with construction trail-blazers such as Laing O'Rourke, Legal and General Modular Homes and Berkeley Modular, along with disruptive innovators such as Sublime and Carbon Dynamic. It shows how the University of Sheffield Advanced Manufacturing Research Centre (AMRC) is collaborating with industry to develop novel techniques and processes that could have significant impact on productivity, quality, training and skills.

\section{Factory flow simulation modelling of a manufacturing facility}

As offsite modular construction moves up the UK government agenda and a stronger market emerges, there will be a shift to fabrication and assembly processes operated within a controlled and equipped work environment before the final modules are delivered to the building site. With the target of delivering greater build efficiencies, build standards and sustainability, modular housing company Berkeley Modular collaborated with AMRC to apply DES techniques to model its proposed new modular manufacturing facility and to conduct a range of factory flow analyses through the model. The project helped to ensure the proposed facility would operate under a lean operational manufacturing environment, which was critical to the government and market insistence on value for money, productivity gains and quality.

DES is a useful analytics tool used to model a set of individual entities moving through a series of queues and activities in a discrete event. The technique has been applied to model complex operational processes, especially within the manufacturing sector, for over a decade to enable a better understanding of 
Civil Engineering

Volume 172 Issue CE6
Using advanced manufacturing technology

for smarter construction

Griffin, Hughes, Freeman et al. operational flow challenges and to validate resource requirements (Liu et al., 2018). Although the concept of applying DES in offsite process management is not a new topic (Alvanchi et al., 2011), there are still limited case studies in the literature of the use of DES in offsite modular construction.

The DES model for Berkeley Modular was developed using Siemens Plant Simulation software. Figure 1 shows a two-dimensional (2D) simulation snapshot of the offsite modular manufacturing site. The simulation model mimics the manufacturing processes from goods in to product out, including raw materials conversion into a range of subassemblies and processes such as painting, drying, fitting and final assembly. The facility supports one-piece flow operation, which becomes a critical requirement for the plant to be able to have as close to $100 \%$ uptime as possible and be able to limit the work in progress to one item at any process.

DES plays an important role to simulate how the operation would work, identify where the key bottlenecks are, and to optimise the planned factory facilities and resources to achieve these targets. As shown in Figure 1, those stations highlighted in yellow are the current bottlenecks within the planned operation due to, for example, limited work stations and variation in cycle times. This information becomes valuable for the company to support its decision-making in reviewing its planned operation and resources.

Overall, the validated DES model helped the company to identify the potential process bottlenecks, validate the required buffer capabilities to maintain lean manufacturing flow and to minimise the requirement for spare resources based on predicted demands. With the help of the DES model, the company has also benefited from further simulation experiments by running a range of what-if scenarios to de-risk changes and validate the impact on key performance indicators, understanding resource utilisation in discrete time.

\section{Immersive construction}

In the past 4 years, consumer-grade immersive VR technologies have become more accessible and cost-effective, creating mass industrial interest in the technology (Moser et al., 2018). Just as companies start to embrace and adopt VR, the advent of AR hardware is changing the visualisation landscape for manufacturing yet again. Although not yet at a consumer-grade quality, the technology presents a huge opportunity to facilitate change across several industries and many are embracing the early-adopter approach to map out the technology.

The AMRC, supported by the Advanced Forming Research Centre and the Warwick Manufacturing Group, ran a project which investigated the wider capabilities of contextualised building information modelling (BIM). BIM is a three-dimensional (3D) model-based process for creating and managing information on a construction project across the project lifecycle. BIM digitally describes every aspect of the built asset, drawing on information accumulated and updated collaboratively at key stages of the project. This digital model provides full visibility of the building's data and leads to more efficient planning, design, construction, and building and infrastructure management.

Building on collaborative research with Glasgow-based visualisation technology specialist Sublime and prefabricated eco-housing manufacturer Carbon Dynamic, the collaboration has extended the capability of an early concept demonstrator produced

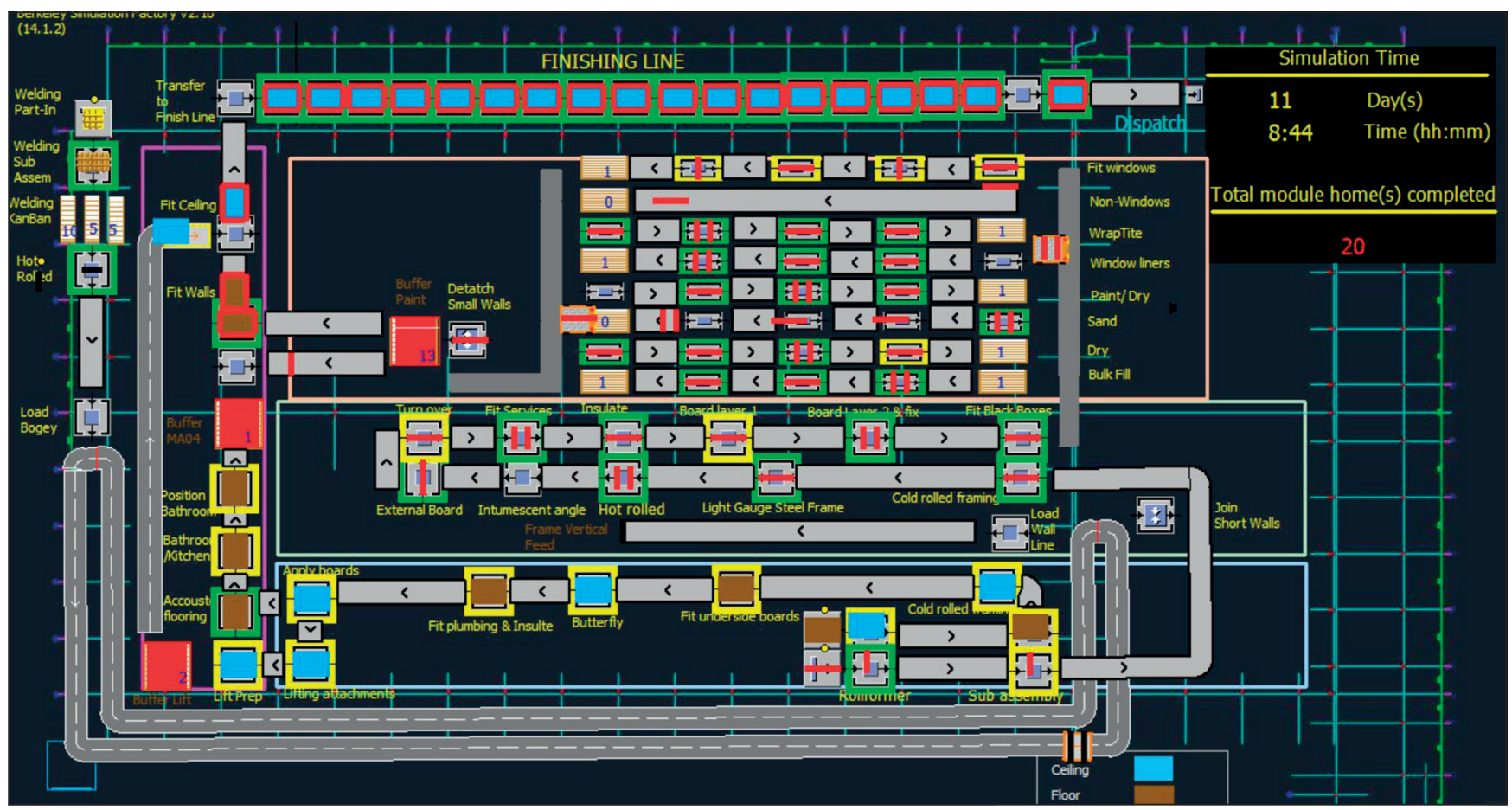

Figure 1. 2D simulation of Berkeley Modular's proposed offsite volumetric factory 
for Crossrail (CIOB, 2015) to demonstrate how AR could assist the manufacturer on its assembly line.

Demonstrators were produced using the Microsoft Hololens headset, which mapped a virtual overlay onto a physical asset (Figure 2). This allowed the manufacturer's workforce to walk around the physical building, seeing the virtual overlay from all aspects while using their hands and speech to interact with the virtual build. A user interface was created giving operators the ability to inspect assets based on a range of identifiers including type, BIM layer and build sequence. To enable this level of detail, both the BIM environment and manufacturing execution system were interrogated, extracting the model and build information, respectively. To demonstrate the value of an AR-enabled pipeline, a live link was also incorporated enabling meta tags, populated in the BIM environment, to be propagated into the AR overlay.

Using the headset's gaze and gesture controls to interact with a physical item, users were able to view all BIM information associated with an item in a virtual information window. This technology improves communication among stakeholders, helps to ensure on-time delivery of construction projects and automates construction management (Ratajczak et al., 2018).

The demonstrator showcased perfectly how a virtual overlay would provide many advantages in an offsite manufacturing environment, not only seeing through solid structures, thus reducing the risk of damaging existing installed components such as wiring and plumbing, but also visualising the corresponding installation plans to help improve quality, prevent rework and increase productivity. The technology also demonstrated how AR could promote a step-change in health and safety in the factory by using the technology to create virtual safety barriers, informing workers of areas that are unsafe to enter without the appropriate personal protective equipment.

The project gave the manufacturer access to the latest tools and technology to identify future opportunities and the barriers they are likely to come across in fully embracing AR. Having complete awareness of both the benefits and barriers has allowed the manufacturer to create a full strategy around the adoption of technology and, more broadly, digital transformation. This activity culminated in the formation of the Augmented Worker (AWE) programme funded by Innovate UK, aspects of which are explored in Section 4.

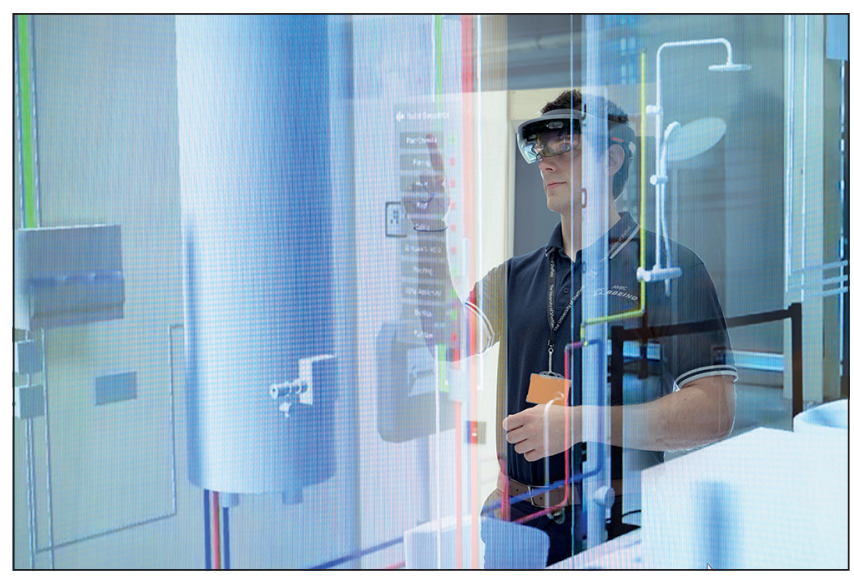

Figure 2. Microsoft Hololens headsets allow users to see a virtual overlay on a physical asset

\section{AWE}

AWE is an advanced interactive platform for the construction industry being developed by AMRC and a consortium of companies under the Innovate UK Innovation Infrastructure Systems round 2 funding (AMRC, 2017). Using AR and VR, the AWE system enables intelligent design, monitoring and maintenance of construction projects by providing a greater level of collaboration and communication between teams and partners on projects. The reduction in the need for paper or mobile-based handheld devices gives workers a hands-free, heads-up solution for a greater level of safety while providing real-time visualisation on site.

The AWE capture system (Gray et al., 2017) allows users to capture and visualise data collaboratively in real time, creating a digital representation of an existing building where no prior digital information was available. It creates a 3D model of the building, identifies assets in the model and labels them with various forms of meta-data. The system can utilise detection hardware to locate services such as plumbing and electric circuits, and record thermal data for an asset.

The benefits the system proposed were, simply, to increase an organisation's awareness of its assets by providing tools to detect and catalogue their location intuitively. This creates impact when considering its use to support operations such as maintenance and servicing: these could be carried out with more precision and with greater speed as they can be planned with a greater understanding of an asset's location and condition when compared with existing methods. Moreover, use of the capture tool provides a mechanism to feed back vital information from ongoing operations.

The various aspects of the asset management solution are discussed in the following sections.

\subsection{Visualisation platform}

The Microsoft Hololens headset was selected as the most appropriate visualisation platform for the application due to its portability and immersive mixed reality capabilities. Its ability to create an overlay enables the user to interact with the virtual and physical world simultaneously. The forward-facing camera, depth sensor and four microphones enable tracking of the capture devices, user hand gestures and voice control.

\subsection{Data capture}

Several devices were selected to work in unison as the environmental capture devices that would build up the digital representation, including a 3D scanner, thermal camera, wall scanner and ground scanner. Two applications were developed using the Unity game engine: the main visualisation platform for the headset and a mobile application to drive data capture on the Asus Zenfone AR. Figure 3 shows an overview of the dataflow through the system between the three components: interface, storage and capture devices.

\subsection{Marker-based tracking}

Vuforia (PTC, Inc., 2018), a software library created specifically for image processing and object detection, was used as a markerbased tracking (Garrido-Jurado et al., 2014) solution for the various capture devices. Using the front-facing camera of the headset, the markers provided a physical location for the datacapture devices used in relation to the headset, enabling the ability 


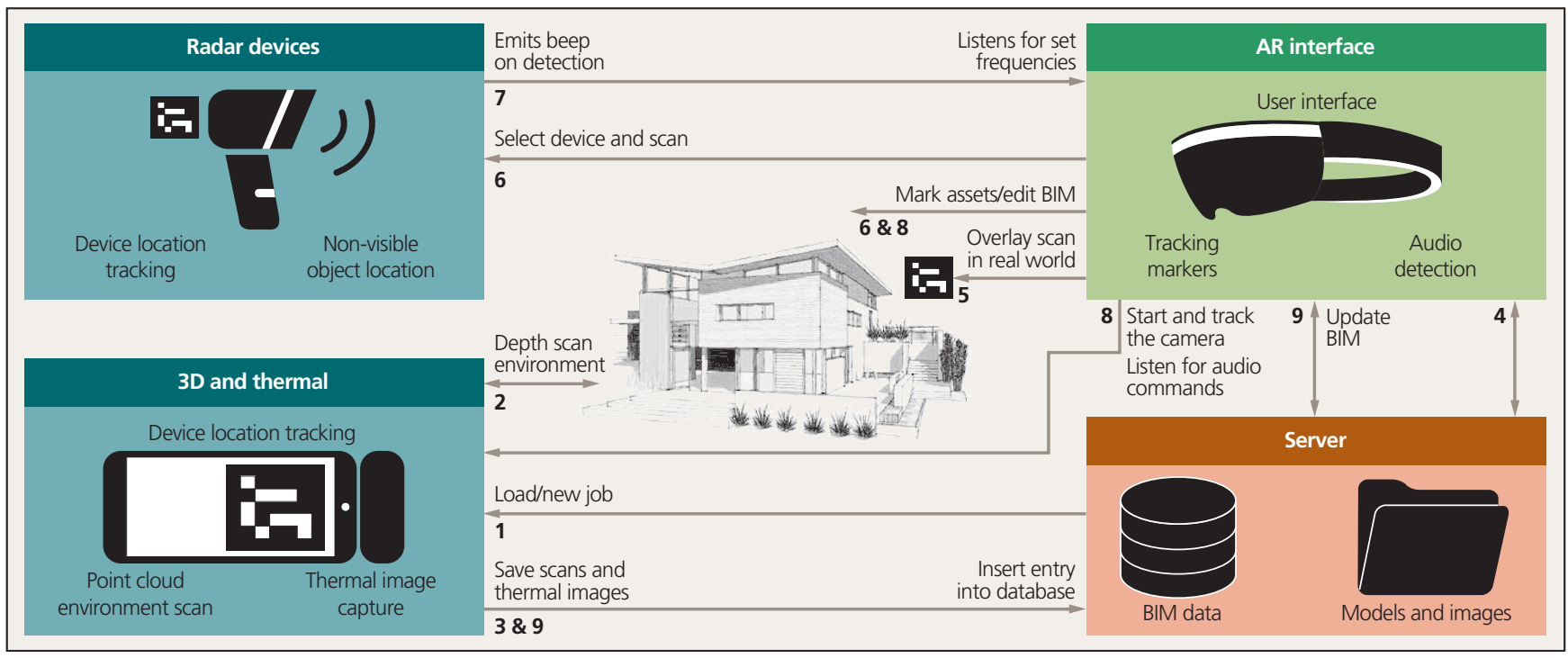

Figure 3. Overview of the dataflow between interface, storage and capture devices of the AWE system

to give spatial references to a detection. Figure 4 shows a capture device displaying a marker.

\subsection{Asset identification}

The asset identification process allows the user to identify an area of the scan as an asset using a bounding box around the physical object. The gesture controls allow the bounding box to be reshaped by pushing and pulling the faces and pulling the edges to rotate. These physical interactive gestures mimic realworld manipulations and provide a level of familiarity to the user. Once an asset has been identified, the user can begin to provide additional data through the use of category fields. By enabling meta tagging while on site, an operative can link data to any asset, creating greater cohesion and providing a more efficient level of cross-referencing information.

\subsection{Device integration}

Integration of the various capture devices proved to be the biggest challenge in creating the asset management system. Most devices were provided with a software development kit or application programming interface, but they were not all provided for the same language or development platform. This resulted in the use of many different communication protocols and the creation of several plug-ins to ensure that the various platforms could communicate. Using audible beeps from the Bosch D-Tect handheld radar scanner it was possible to detect accurately objects with minimal dropped detections. By limiting the frequency and decibels, it was possible to filter out the background noise and eliminate false detections in a moderately noisy manufacturing environment. However, in noisier environments the headset's microphones can struggle to detect the beeps.

\subsection{BIM data storage and collaboration}

The current asset management solution uses a structuredquery-language database to store the information created from annotating a scan. Although not being stored in a recognised BIM

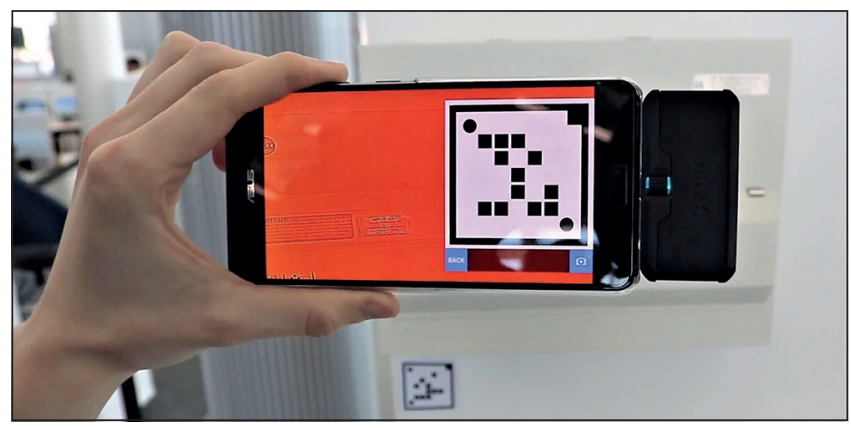

Figure 4. An example of spatial reference marker displayed on a capture device

file format, the data set was constructed to be easily converted to Industry Foundation Classes format. Using the xBIM toolkit (xBIM, 2018), the asset management system would be able to store the data captured in a format that would allow the BIM data to be compatible with popular BIM management software, such as Autodesk Revit, and would adhere to various countries' standards for government-aided construction projects, including the Danish, Finnish and Norwegian governments. By using a single copy of the data in a cloud solution, and with real-time updates as scans are created and converted into BIM data, all users of the asset management system can be assured they are viewing the most upto-date version information. The asset management system can also be accessed and modified by multiple users at once, enabling highly scalable, on-site or remote collaboration.

\subsection{Impact}

Figure 5 shows the output of the headset combined with the wall scanner. These digital technologies provide a method for intelligently managing assets without associated BIM information, and will dramatically reduce energy wastage and lower overall costs. 
Civil Engineering

Volume 172 Issue CE6
Using advanced manufacturing technology

for smarter construction

Griffin, Hughes, Freeman et al.

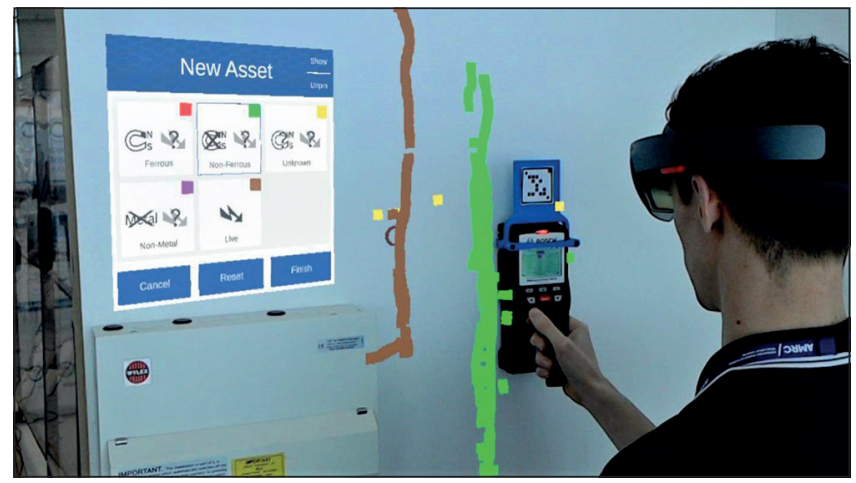

Figure 5. An example of data capture with a Microsoft Hololens headset and a Bosch D-Tect handheld wall scanner

\section{Automated construction demonstrator}

At the time of writing the authors have not found any direct research on light-gauge steel framing (LGSF) assembly. However, similar work has been undertaken for the assembly of timber-frame trusses and automated fastening of steel components (Malik et al., 2018; Trussmatic Oy, 2019). Manually manufacturing a steel stud wall panel requires multiple workers and is a time-consuming, dextrous process, which includes tasks that are repeated numerous times over the course of the build.

To show the art of the possible in the automation of traditional processes, AMRC created an automated robotic demonstrator capable of assembling a full-scale $2 \times 3 \mathrm{~m} 2 \mathrm{D}$ stud wall panel made from LGSF C-sections. The robotic cell was capable of picking and placing the sections into position and applying mechanical fixings. Figure 6(a) shows a digital model of the robot cell and Figure 6(b) shows the robot in action. An ABB IRB 6700-235 robot was chosen as it has a $2.65 \mathrm{~m}$ reach, enabling full-size components to be processed.

A flexible fixture was required to build different designs of LGSF stud walls. A novel modular, reconfigurable fixture, which consisted of 'pegboard'-inspired features was conceptualised by AMRC and manufactured by Metlase. The concept for this pegboard is to have a modular fixture with a series of holes in a fixed pitch for movable component interfaces. The component interfaces can be placed anywhere within the fixture to make datum positions. The interfaces are used as a datum interface for the LGSF.

To have the ability to pick and place the pegboard interfaces and sections, automatic grippers are required. AMRC designed and built a robot end effector using dual grippers with adjustable jaws for flexibility. The end effector consists of two Zimmer IO Link electric grippers. Electric grippers were chosen for this task over common pneumatic grippers, as the gripper gives jaw force and positional data enabling a feedback loop to be introduced with statuses known. This is in contrast to pneumatic grippers, which are simply open or closed without additional feedback, which could potentially damage the sections.

A pneumatically controlled Gesipa Taurus 2 riveter was used to complete the assembly of the stud wall by mechanically fixing the parts together. The robot moves to positions where a rivet is required and the tool inserts the rivet in position, then draws the mandrel through the tool, fixing the rivet securely in position.

The demonstrator showed comparable process times to that of a team of two to three operators (Howick, 2019) but repeatability and hence quality was improved. It should be noted that a robotic cell can operate continuously without intervention, assuming material supply is available. There are also benefits relating to health and safety of the operators due to reduced manual-handling risks. The flexibility of this automation and fixture also enabled higher product variation over more traditional automation.

Given the short time frame in which the system was developed, further optimisation of the robotic process could lead to further process time improvements. Further work could be undertaken to realise the full benefits of this system, such as version control driven from component models, assembly data for robotic operations accessed from computer-aided design, and placing the assembly system next to a traditional cold-roll-forming machine.

\section{Inspection for modular construction}

Reality-capture devices such as 3D laser scanners and highresolution imaging systems have been used in the construction industry for more than a decade (Boukamp and Akinci, 2007; Gordon et al., 2003; Hajian and Becerik-Gerber, 2009; Su et al., 2006). However, acceptable tolerances within the traditional construction sector are large when compared with the aerospace

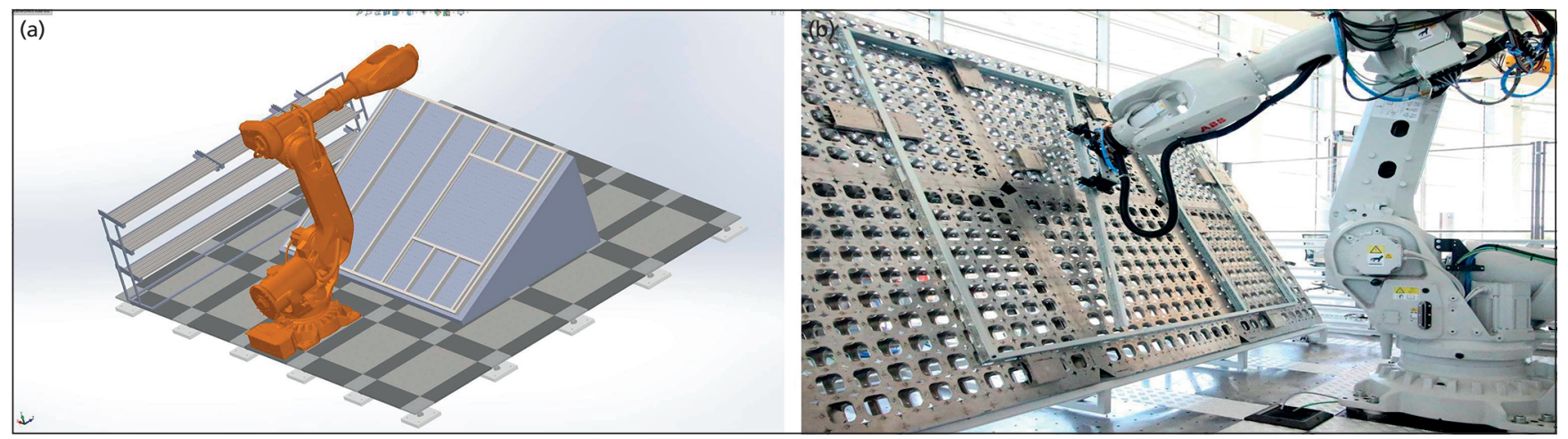

Figure 6. Digital model of a robot cell for assembling light-gauge steel framing (a) and robot placing C-sections on a pegboard (b) 
Civil Engineering

Volume 172 Issue CE6
Using advanced manufacturing technology

for smarter construction

Griffin, Hughes, Freeman et al. industry (i.e. millimetres compared to micrometres). New automated production processes and the modular build philosophy have necessitated a reduction in allowable product variation, which means that inspection plays a larger role in the production process to ensure product quality.

For housing, one construction method is to use cross-laminated timber (CLT) in large panels, which are assembled to create a volumetric module. The CLT panels often have computer numerical control machined features. To avoid stack-up of tolerances in the final assembly, measurement data are required to ensure that the features are within tolerance.

Legal and General Modular Homes provided AMRC with a sample CLT panel that contained features typical of a modular house wall. The panel was $4.5 \times 2.5 \mathrm{~m}$ and manufactured from $100 \mathrm{~mm}$ CLT. AMRC investigated two methods of feature assessment. The first used a 3D laser scanner to capture the complete 3D geometry of the component, which can then be used to perform a dimensional assessment. The second method utilised machine vision, with a pan-tilt-zoom camera to provide component assessment based on images.

\subsection{D geometry assessment}

AMRC developed an automated scanning system which utilises a Surphaser 25HSX to capture high-resolution point cloud data. The spherical scanner uses a rotating mirror to capture up to 1.2 million points per second over a range of up to $30 \mathrm{~m}$ (Figure 7). An automated scanning and analysis procedure was created using InnovMetric PolyWorks. For this application the resolution was reduced to approximately $0.9 \mathrm{~mm}$ point spacing at $3 \mathrm{~m}$, which required a scan time of approximately $4 \mathrm{~min}$. The captured measurement information was aligned and compared to the computer-aided design and the output displayed as a colour heat map showing the deviation from nominal (Figure 8(a)).

As the scanner is a line-of-sight instrument, it can only capture features which have an unobstructed view to the sensor. This means that in this application, the scanner is only able to assess the top surface of the panel; panel thickness cannot be measured without additional tooling. AMRC is conducting further research to understand the challenges with deploying this technology for volumetric inspections, such as for complete modules.

The current 'first-article inspection' process is very manual and typically takes in excess of $2 \mathrm{~h}$, meaning the proposed

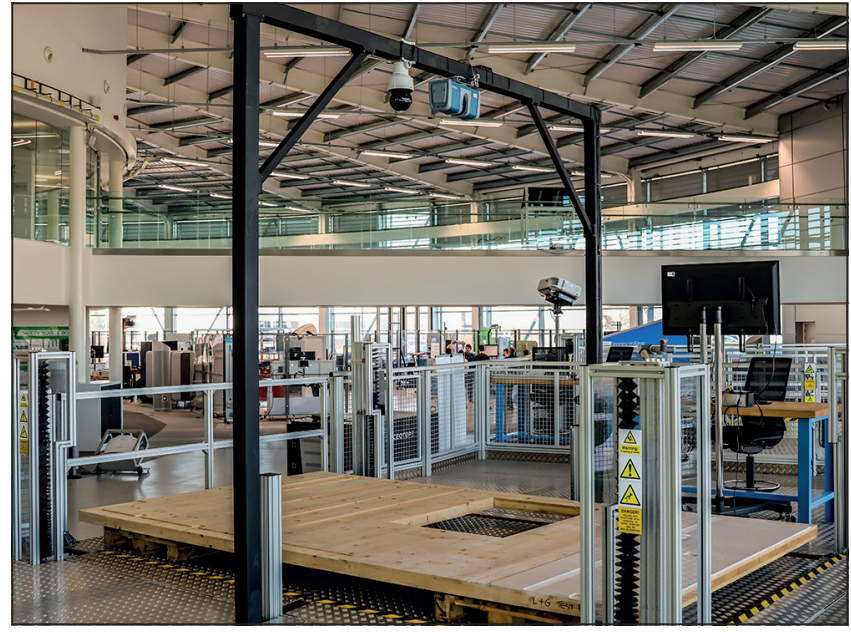

Figure 7. Inspection cell for checking a CLT wall panel using a 3D laser scanner

system represents a significant time saving. In addition to this, the amount of information captured is significantly greater than current methods, meaning it is possible to achieve a much greater understanding of the manufacturing process and therefore apply methods such as statistical process control to improve product quality.

\subsection{D image assessment}

Currently, visual checks are performed by human operators to assess a component or assembly quickly to ensure all required features are present and there are not any manufacturing defects. This process is prone to error, especially on large assemblies where the number of inspection checks required is large.

Machine vision systems are high-quality cameras paired with intelligent software and are used widely in manufacturing sectors such as pharmaceuticals, food and drink and consumer electronics. Typically these systems use a camera or set of cameras with a fixed field of view. It was found, however, that for this application at least six fixed cameras would be required to capture a complete panel and this would not provide a suitable pixel/millimetre ratio at a working distance of $3 \mathrm{~m}$. Instead, AMRC investigated
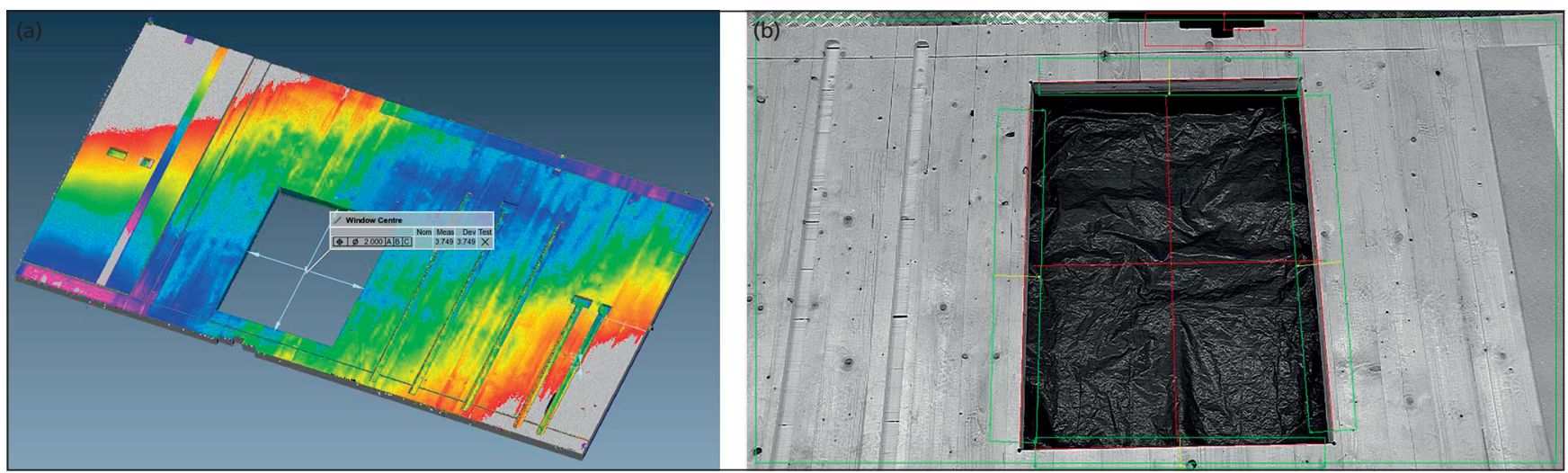

Figure 8. Colour heat map of scanned deviations in the timber wall panel (a) and image assessment to check opening positions (b) 
Civil Engineering

Volume 172 Issue CE6
Using advanced manufacturing technology

for smarter construction

Griffin, Hughes, Freeman et al. the use of a pan-tilt-zoom camera, which allows a much larger working volume as the camera can be reoriented to capture areas of interest. In addition, the zoom function means an acceptable pixel/ millimetre ratio can be achieved.

The camera chosen was an Axis Q6115-E, a high-end closedcircuit television camera that has 1080 pixel resolution and up to 30 times optical zoom. AMRC developed a control system and image-analysis routine using Labview (Figure 8(b)). For the demonstration, the system captured and analysed ten images of key areas in approximately $1 \mathrm{~min}$. The developed system was shown capable of inspecting CLT panels for common defects, such as incorrect placement of components and feature positioning. A calibration method was devised to allow for direct measurement of individual features, such as slots and gaps. Baseline measurements were taken with a vernier calliper and the image was found to be within $\pm 0.3 \mathrm{~mm}$.

The technology was demonstrated on an individual component but it is likely to have large benefits when used for assessing assemblies. Given the quick capture time of the system, it could be used during first and second fix to capture any manufacturing defects in near real time. It was demonstrated that an aerospacegrade 3D scanning system is capable of inspecting large-scale construction components and that a calibrated pan-tilt-zoom camera can be used for part inspection, which greatly improves the current process of visual and manual checking.

\section{Conclusion}

This paper demonstrates the potential of integrating emerging technologies and techniques such as robotics and automation, AR and VR, DES, large volume metrology, and improved tools and processes within the construction field in an efficient and effective manner.

Mature technologies from other sectors can, through horizontal innovation, be directly transferred into a new sector to solve similar industry challenges. Significant productivity increases are possible, with the benefit often driven by the digitalisation of traditionally manual paper-based processes.

The technologies can be applied to construction manufacturing processes. The direct use of digital information from the digital model also reduces the chances of error introduced by using paper drawings and manual techniques and will ultimately lead to quality improvements. The benefits demonstrated by these individual technologies will be additive when combined into an integrated digital manufacturing framework.

Substantial research efforts are still required across the sector to utilise these emerging technologies fully to show an impact. There also needs to be a change of mindset in the construction industry to adopt these technology research outcomes into production.

\section{References}

Alvanchi A, Aximi R, Lee S and AbouRizk SM (2011) Offsite construction planning using discrete event simulation. Journal of Architectural Engineering 18(2): 114-122.

AMRC (Advanced Manufacturing Research Centre) (2017) Soluis Group Secures 11 Million Innovate UK Grant for the Construction Industry. See https://www.amrc.co.uk/news/soluis-group-secures-1-million-innovate-ukgrant-for-the-construction-industry (accessed 25/11/2018).
Brown M (2018) Time to Modernise - Exclusive Interview with Mark Farmer See https://www.ukconstructionmedia.co.uk/news/time-to-moderniseexclusive-interview-with-mark-farmer/ (accessed 25/11/2018).

Boukamp F and Akinci B (2007) Automated processing of construction specifications to support inspection and quality control. Automation in Construction 17(1): 90-106.

CIOB (Chartered Institute of Building) (2015) Soluis and Crossrail to Test Smart Helmets on Site in Innovate UK Trial. See http://www.bimplus. co.uk/news/soluis-a3nd-cros6srail-test-smart-helm2ets-site/ (accessed 25/11/2018)

Farmer M (2016) The Farmer Review of the UK Construction Labour Model: Modernise or Die. Construction Leadership Council, London, UK.

Garrido-Jurado S, Muñoz-Salinas R, Madrid-Cuevas FJ and Marín-Jiménez MJ (2014) Automatic generation and detection of highly reliable fiducial markers under occlusion. Pattern Recognition 47(6): 2280-2292.

Gordon C, Boukamp F, Huber D et al. (2003) Combining reality capture technologies for construction defect detection: a case study. EIA9: E-Activities and Intelligent Support in Design and the Built Environment, 9th EuroplA International Conference, Istanbul, Turkey, pp. 99-108.

Gray J, Chivaura A and Frias C (2017) Augmented Worker - Capture. Advanced Manufacturing Research Centre with Boeing, Sheffield, UK.

Hajian H and Becerik-Gerber B (2009) A research outlook for real-time project information management by integrating advanced field data acquisition systems and building information modeling. In Computing in Civil Engineering (2009) (Caldas CH and O'Brien WJ (eds)).

HM Treasury (2017) Autumn Budget 2017. See https://www.gov.uk/ government/publications/autumn-budget-2017-documents/autumnbudget-2017 (accessed 25/11/2018).

HMG (Her Majesty's Government) (2018) Industrial Strategy - Construction Sector Deal. The Stationery Office, London, UK.

Howick (2019) Bathroom Pods. See https://www.howickltd.com/applications/ modular-construction/bathroom-pods/ (accessed 12/03/2019).

IPA (Infrastructure and Projects Authority) (2016) Government Construction Strategy 2016-20. IPA, London, UK

IPA (2017) Transforming Infrastructure Performance. IPA, London, UK.

Liu R, Xie X, Yu K and Hu Q (2018) A survey on simulation optimization for the manufacturing system operation. International Journal of Modelling and Simulation 38(2): 116-127

Malik N, Ahmad R and Al-Hussein M (2018) Generation of safe tool-paths for automatic manufacturing of light gauge steel panels in residential construction. Automation in Construction 98(2): 46-60.

Moser T, Hohlagschwandtner M, Kormann-Hainzl G, Pölzlbauer S and Wolfartsberger J (2018) Mixed reality applications in industry: challenges and research areas. In Software Quality: The Complexity and Challenges of Software Engineering and Software Quality in the Cloud. SWQD 2019 (Winkler D, Biffl S and Bergsmann J (eds)). Springer, Berlin, Germany, vol. 338, pp. 95-105.

PTC, Inc. (2018) Vufori Augmented Reality. See https://www.vuforia.com/ (accessed 12/03/2019)

Ratajczak J, Schweigkofler A, Riedl M and Matt DT (2018) Augmented reality combined with location based management system to improve the construction process, quality control and information flow. In Advances in Informatics and Computing in Civil and Construction Engineering (Mutis I and Hartmann T (eds)). Springer, Berlin, Germany, pp. 289-296.

SCST (Science and Technology Select Committee) (2018) Offsite Manufacture for Construction: Building for Change. See https:// publications. parliament.uk/pa/ld201719/ldselect/ldsctech/169/16902.htm (accessed 25/11/2018).

Su YY, Hashash YMA and Liu LY (2006) Integration of construction as-built data via laser scanning with geotechnical monitoring of urban excavation. Journal of Construction Engineering and Management 132(12): 1234-1241.

Trussmatic Oy (2019) Smart Robotic Solutions: Fully Automated Roof Truss Line. Trussmatic Oy, Helsinki, Finland. See https://www.trussmatic.com/ smart-robotic-solutions/ (accessed 12/03/2019).

XBIM (2018) XBIM Toolkit. See http://docs.xbim.net/ (accessed 25/11/2018). 